Cliffs. The aim of the Museum's project is to verify the age of the carvings-generally thought to be several hundred years old - and to try to establish correlations between age and style for Tellem and Dogon wood carvings. The radiocarbon tests are carried out by Isotopes, Inc., U.S.A., and a first series is in progress on the Museum's own objects. A second series is planned for which the Museum hopes to have the collaboration of other institutions whose collections contain Dogon and Tellem material. Publication of the results is planned after completion of the entire project (i.e. no individual results will be published).

\title{
Un petit musée de brousse à Bangassou
}

L'EsT de la République Centrafricaine et le nord du Congo ont été jadis le siège d'une très vieille civilisation, la civilisation nzakara/zandé, qui a survécu jusqu'à nos jours, plus ou moins affectée par le cours des affaires du monde. Près de la capitale de l'ancien royaume de Bangassou, deux sociologues, avec l'encouragement de l'inspecteur de l'enseignement, ont créé un petit musée de brousse où ils ont rassemblé quelques objets anciens sauvés du feu, de l'eau et des termites.

Pourquoi un musée en brousse? Simplement pour pouvoir offrir sur place, aux enfants des écoles, à la jeunesse questionneuse, un témoignage authentique et concret de leur passé glorieux. Une collecte étonnament homogène d'objets usuels anciens, de belles armes, de faible valeur marchande, souvent rares et tous de bonne forme a été recueillie au pays nzakara. Une autre est en cours de rassemblement, consacrée au pays zandé; les deux d'ailleurs sont parentes. Des copies de documents historiques, des cartes originales retraçant l'histoire ou expliquant l'organisation très sociale du pays complètent les collections.

Le tout est conservé dans une salle de la Mission sociologique du Haut-Oubangui (B.P. 68, Bangassou R. C. A.), pour l'instant encore dans des caisses der fer, faute de moyens muséographiques. Les mesures de conservation appropriées ont été prises, inventaire et photographies peuvent être communiqués. On voit comment un tel effort peut contribuer à forger l'unité nationale.

\section{(Communication de Monsieur E. de Dampierre)}

\section{INADES: Correspondence Courses in Sociology and Economics}

INADES (Institut Africain pour le Développement Économique et Social, B.P. 8008, Abidjan, Côte d'Ivoire) has produced a series of courses for adult education by correspondence. The cost of each course is $30 \mathrm{frs}$. (20 frs. without homework) or the equivalent in other currencies. The following courses are now available:

Cours de sociologie, by R. Bureau.

Sociologie générale. Cours d'introduction; sociologie familiale et démographie sociale; sociologie religieuse; sociologie politique et juridique; sociologie du travail; sociologie urbaine; psychologie sociale; la société moderne, les changements sociaux; conclusions: l'intégration sociale. Personne et société.

Économie politique, by Xavier Baronnet.

Découvert de l'économie; le circuit économique; aspects géographiques de l'économie; la population; la production des richesses; le travail; le capital, l'entreprise; la répartition des richesses: prix et marchés, salaires, profits; l'utilisation des richesses: la monnaie, le crédit, consommation et épargne; fluctuation et maîtrise de la vie économique.

Développement et planification, by R. Houi and J.-L. Fyot.

Conditions géographiques du développement; conditions économiques; conditions sociologiques; un cas non-africain: l'Inde; un cas africain: les pays de savane; les problèmes de la croissance; le modèle de croissance; l'élaboration du plan. 